3. Mathews SC, Pronovost PJ. Physician autonomy and informed decision making. Finding the balance for patient safety and quality. JAMA 2008;300:2913-15.

4. Mills PD, Neilly J, Kinney LM, et al. Effective interventions and implementation strategies to reduce adverse drug events in the Veterans Affairs (VA) system. Qual Saf Health Care 2008;17:37-46.

5. Wachter RM, Flanders SA, Fee C, et al. Public reporting of antibiotic timing in patients with pneumonia: lessons from a flawed performance measure. Ann Intern Med 2008;149:29-32.

6. Amalberti R, Auroy Y, Berwick D, et al. Five system barriers to achieving ultrasafe health care. Ann Intern Med 2005;142:756-64.

\title{
Market-based control: the solution to slow progress with patient safety?
}

\author{
Richard G Thomson
}

In this issue of Quality and Safety in Health Care, there is a paper that should stimulate considerable debate (see page 99); ${ }^{1}$ indeed, we have published three commentaries alongside the paper ${ }^{2-4}$ to initiate this and a further response from the authors (see page 90). ${ }^{5}$ Despite major initiatives to improve patient safety, there is a perception that attempts to improve safety have made slow progress. Hence, Coiera and Braithwaite argue for the implementation of market based control mechanisms as an incentive to promote patient safety. Their proposal is modelled on the "cap and trade" approach to creating a market in emissions trading, a key component of the Kyoto protocol that allows organisations that are successful in reducing carbon emissions to sell credits to organisations that have been less successful.

The parallel between emissions trading to improve the environment and patient safety event trading to improve healthcare safety is fascinating; each of our commentators is intrigued by the proposal. However, each of them believes that the model, while intriguing, is unlikely to be implemented or effective. Coiera and Braithwaite have responded to these commentaries with a further robust argument!

Chin and Wilkes ${ }^{2}$ are concerned that a market of this type will lead to further widening of inequalities through a variety of mechanisms including lobbying power, resource constraints, ability to pass or shift costs and gaming. If market mechanisms were to enhance inequalities, this would be unacceptable (see page 88 ). While Chin and Wilkes believe that measurement of patient safety and holding

Correspondence to: Professor Richard G Thomson, Department of Epidemiology and Public Health, School of Health Sciences, Medical School, Framlington Place,

Newcastle upon Tyne NE2 4HH, UK;

richard.thomson@newcastle.ac.uk healthcare organisations accountable in a fair manner, consistent with Coiera and Braithwaite's proposals, are important, neither is unique to market solutions.

Donaldson $^{3}$ expresses a fundamental concern-he argues that healthcare is not a public good in the same way as the environment (see page 87). He also points out that the introduction of quasimarkets in healthcare has been largely unsuccessful in addressing issues of quality and safety. Instead he calls for more explicit and better developed methods to determine priorities for investment in constrained healthcare systems. He also, quite rightly, raises the question as to whether the emissions trading model has yet shown itself to be effectiveindeed, Coiera and Braithwaite themselves accept that it is too early to evaluate that.

Meltzer ${ }^{4}$ points out that incentives for patients and payers to avoid errors through competitive market forces already exist, in contrast to carbon emissions prior to trading, thus making the argument less compelling (see page 86). He highlights the challenge of measurement and of how an appropriate level of adverse events might be set. He also believes that such a system is likely to increase healthcare costs. Meltzer flags up one element of the proposal that he describes as compelling; that is the underlying assumption that some level of harm is appropriate or acceptable because reducing harm is costly.

In addition to the concerns expressed in these commentaries, I believe there are several other issues that need to be considered before pursuing an MBC approach. First, this approach is very top down; it appears to ignore the importance of engagement of healthcare staff in improving safety. The mantra of "first do no harm" is embedded within the culture of most healthcare professionals, and when patient safety incidents occur, they are rarely due to negligence or intended actions but largely reflect the inevitabilities of human error and the inadequacies of systems. A top-down model such as that proposed here is likely to provoke resistance among professional groups.

It is also likely to provoke resistance among patients and the public. What level of acceptability would this engender within the public domain, particularly given Meltzer's comments that an underlying implication is that there is a level of acceptable harm? One of the challenges to patient safety has been the fact that the value placed upon harm produced by healthcare is often quite different to the value placed upon injury or ill health arising de novo. Fundamentally, patients access healthcare with the expectation that it will make them better and find the concept that it might make them worse very difficult to understand.

Another issue of relevance is the complexity of healthcare. The issue of carbon emission is arguably much more straightforward in both its measurement and its aetiology than harm caused by healthcare. This complexity in healthcare may explain why some of the methods of quality and safety improvement that have been effective in industrial settings are more difficult to apply in healthcare. Market-based control is likely to be similar in this respect.

A key problem, also flagged up by our commentators, is that of measurement. We know that incident reporting significantly under-reports for a variety of reasons. Equally, there is evidence to suggest that those organisations that report more incidents have a better and more effective safety culture. ${ }^{6}$ Any market-based mechanism that penalised higher rates of incidents would have the potential effect of switching off the tap of reporting, upon which much safety improvement depends. The approach would be replete with perverse incentives. Furthermore, the use of measures of safety or quality from routine information systems, such as the AHRO indicators suggested by Coiera and Braithwaite, would need to take account of the fact that routine data quality and completeness are hugely variable, not only across 
different healthcare systems but also within countries, again emphasising the considerable challenge of measurement of safety within this proposal.

In conclusion, this is a fascinating and intriguing proposal that merits wide debate. But is this proposal something that healthcare systems should take seriously and consider testing?

Could this approach dramatically improve safety within healthcare systems, or should we once again heed $\mathrm{HL}$
Mencken's view that "For every complex problem there is an answer that is clear, simple, and wrong"?

\section{Competing interests: None.}

Qual Saf Health Care 2009;18:83-84. doi:10.1136/qshc.2008.030379

\section{REFERENCES}

1. Coiera $\mathbf{E}$, Braithwaite J. Market-based control mechanisms for patient safety. Qual Saf Health Care 2009;18:99-103.
2. Chin MH, Wilkes AE. Equity concerns in market-based control schemes for patient safety. Qual Saf Health Care 2009; 18:88-90.

3. Donaldson C. Is safety safe in the market's hands? Qual Saf Health Care 2009;18:87-8.

4. Meltzer D. The Coase Theorem and patient safety. Qual Saf Health Care 2009;18:86-7.

5. Coiera E, Braithwaite J. Debating market-based control for patient safety: response to commentaries. Qual Saf Health Care 2009;18:90-1.

6. Hutchinson A, Young TA, Cooper KL, et al. Trends in healthcare incident reporting and relationship to safety and quality data in acute hospitals: results from the National Reporting and Learning System. Qual Saf Health Care 2009;18:5-10

\section{The dangers of heparin flushes}

\section{Brian Toft}

Heparin is undoubtedly a useful medicine in the battle against ill-health. However, besides being used for prophylaxis, heparin is also widely used to flush intravenous peripheral and central lines in an attempt to keep them patent.

\section{DANGERS ASSOCIATED WITH THE USE OF HEPARIN FLUSHES}

There is evidence to suggest that heparin flushes are a serious risk to patients without providing any commensurate benefits. For example, at a London hospital, vials containing several doses of the medicine were on two separate occasions administered in their entirety to two different patients. This resulted in the patients receiving 25000 IU of heparin rather than the intended dose of 5000 IU and requiring medical intervention to remedy the situation. Fortunately neither incident resulted in any long-term harmful effects. However, on another occasion this same error in the same hospital "resulted in death from cerebral haemorrhage". 1

More recently in the UK, four young patients were admitted to an acute trust's day bed unit to undergo diagnostic tests. As young children do not tolerate invasive procedures very well when awake, the tests were to be carried out under general anaesthesia. As part of the procedure the consultant paediatric anaesthetist was to administer a heparin flush. However, owing to human error and systems failures, each child was inadvertently administered 25000 IU of heparin-that is, a

Correspondence to: Brian Toft, 1 Gartons Road, Middleleaze, Swindon SN5 5TS, UK; brian.toft@ ntlworld.com dose 500 times greater than that which had been intended. None of the children involved in these incidents were reported to have experienced any lasting effects. However, the seriousness of these events cannot be overstated. Indeed, as a direct result of the author's report ${ }^{2}$ into these four patient safety incidents (PSIs) the UK's National Patient Safety Agency (NPSA) issued a "Rapid Response Report", warning the medical profession of the risk associated with heparin flushes. ${ }^{3}$

\section{EFFICACY OF HEPARIN FLUSHES}

The PSIs noted above illustrate that heparin flushes can carry considerable risk for patients. However, there does not seem to be any evidence that heparin flushes are more beneficial in keeping intravenous catheters and cannulas patent than flushing them with saline, particularly in adults. ${ }^{4}$

One of the reasons for the controversy regarding the efficacy of heparin flushes, particularly with regard to children, is the lack of research in this area. There are of course major ethical dilemmas in carrying out such studies on children and therefore the reluctance of the medical profession to pursue them is understandable. However, two studies in The Cochrane Central Register of Controlled Trials, one in neonates $^{5}$ and the other in the paediatric population, ${ }^{6}$ came to the conclusion that saline was just as effective as heparin in maintaining the patency of peripheral lines using cannulas. Another study on the "Treatment of catheter occlusion in paediatric patients" came to a similar conclusion.

With respect to the use of heparin flushes to maintain patency in central venous and arterial catheters the evidence is more problematic. Thus further research is urgently needed to clarify whether the benefits of using a heparin flush outweigh the risks, particularly with respect to children.

\section{VERBAL DOUBLE-CHECKING SAFETY PROTOCOLS}

Although "Human error is inevitable...", the Chief Medical Officer for England argues that "harm to patients is not". ${ }^{8}$ For example, if an inadvertent error is made by a healthcare professional but prevented from reaching the patient by a colleague then although an error was made no harm will have occurred-that is, a near-miss will have taken place as opposed to an iatrogenic event.

One potential way to reduce the risk of patients being harmed in healthcare settings is through the use of an explicit, appropriately configured, verbal doublechecking safety protocol. For example, the Human Fertilisation and Embryology Authority has made such protocols (known as "witnessing") mandatory in the UK. ' This is because such protocols can help identify inadvertent human errors and thus reduce the risks to patients. ${ }^{10}$ Similarly, it is considered best practice that prior to the administration of radiotherapy a verbal double-checking safety protocol of each patient's treatment parameters is undertaken by the radiographers.

Frequently, analogies are drawn between the medical profession and the commercial aviation industry. However, one major difference between the two professions, in terms of their operating procedures, is that whereas all aircrews must undertake verbal double-checking safety protocols, members of the medical profession do not. The UK commercial aviation industry has an excellent record for safety. For example, the UK Civil Aviation Authority has recently stated that ${ }^{11}$. 